\section{A Hamilton-Jacobi Setup for the Static Output Feedback Stabilization of Nonlinear Systems}

\author{
Alessandro Astolfi and Patrizio Colaneri
}

\begin{abstract}
The (local) static output feedback stabilization problem for a class of nonlinear (affine) systems is discussed. A sufficient condition is established and a (partial) converse is worked out. This condition provides a counterpart to a well-known result of linear systems theory. The effectiveness of the developed theory is illustrated with a simple example.
\end{abstract}

Index Terms-Hamilton-Jacobi equations, nonlinear systems, static output feedback.

\section{INTRODUCTION}

The static output feedback (SOF) stabilization problem consists in finding, for a given input-output control system, a static output feedback control law such that the resulting closed-loop system is asymptotically stable. Despite the simplicity of this statement and the great amount of literature, the SOF problem is still one of the most celebrated open issues in systems and control.

This problem is important in its own right, since static output feedback controllers are less expensive to implement and (possibly) more reliable in practice. In particular, static output feedback controllers can be employed as back-up controllers, i.e., very simple controllers which are not active during the regular operation of the plant, but which are used in case of faults.

Finally, it is easy to show that the design of dynamic output feedback controllers (or of asymptotic state observers) boils down to the solution of a static output feedback control problem for an augmented system.

The SOF stabilization problem for linear systems has been widely studied; see [12], the references therein, and the recent contributions [7], [6], [8], [10], [13], and [2]. Note however that, even in the case of linear systems, several problems are still open.

The SOF stabilization problem for nonlinear systems has not been studied with similar interest and, to the best of the authors's knowledge, no general result is available. However, several results have been developed for nonlinear passive systems, see, e.g., [4] and [5].

This note tries to produce a step forward in the understanding of the local SOF stabilization problem for nonlinear systems, providing a sufficient condition, together with a partial converse, which constitute the counterpart, mutatis mutandis, of well-known conditions for linear systems.

The note is organized as follows. In Section II, the problem is formally stated, whereas in Section III the sufficient and the necessary conditions are provided in terms of the solution of a constrained Hamilton-Jacobi equation along with a rank condition. Finally, Sections IV and V contain a simple illustrative example, some comments and hints for future research.

Manuscript received July 18, 2001; revised February 20, 2002. Recommended by Associate Editor H. Wang. This work was supported in part by the TMR Network NACO2.

A. Astolfi is with the Dipartimento di Elettronica e Informazione, Politecnico di Milano, 20133 Milano, Italy, and also with the Department of Electrical and Electronic Engineering, Imperial College, London SW7 2BT, U.K. (e-mail: a.astolfi@ic.ac.uk).

P. Colaneri is with the Dipartimento di Elettronica e Informazione, Politecnico di Milano, 20133 Milano, Italy (e-mail: colaneri@elet.polimi.it).

Digital Object Identifier 10.1109/TAC.2002.805680

\section{PRELIMINARIES}

Consider the nonlinear (affine) system

$$
\begin{aligned}
& \dot{x}=f(x)+g(x) u \\
& y=h(x)
\end{aligned}
$$

where $x \in \mathbb{R}^{n}$ denotes the state of the system, $u \in \mathbb{R}^{m}$ the control input, $y \in \mathbb{R}^{p}$ the measured output, and the mappings $f(x), g(x)$ and $h(x)$ are smooth mappings defined in a neighborhood of the origin of $\mathbb{R}^{n}$. Moreover, without loss of generality, we also assume that $x=0$ is an equilibrium point, i.e., $f(0)=0$ and $h(0)=0$.

The local static output feedback stabilization problem for (1) and (2) consists in finding, if possible, a static control law described by

$$
u=F(y)
$$

such that the origin is a locally asymptotically stable equilibrium of the closed-loop system. If such an output feedback does exist, we say that (1) and (2) is SOF stabilizable and that $F(\cdot)$ is a solution of the SOF stabilization problem.

Finally, to describe the main results of this note, we introduce the following definitions.

Definition 1: The pair $\{f, h\}$ is said to be locally detectable (observable) if there exists a neighborhood $U$ of the point $x=0$ such that, if $x(t)$ is any integral curve of $\dot{x}=f(x)$ satisfying $x(0) \in U$, then $h(x(t))$ is defined for all $t \geq 0$ and $h(x(t))=0$, for all $t \geq 0$ implies $\lim _{t \rightarrow \infty} x(t)=0(x(t)=0$, for all $t \geq 0)$.

Definition 2: Given a smooth mapping $y=h(x)$, we denote with ${ }^{1}$ $\operatorname{ker}(h)$ the set of all $x$ such that $y=0$.

\section{Static OutPut FeEdback Stabilization FOR NONLINEAR SYSTEMS}

This section contains the main results of the note, namely two sufficient conditions for the solution of the local SOF stabilization problem for nonlinear systems and a partial converse. It is also shown that the sufficient conditions, once specialized to the case of linear systems, coincide with well-known sufficient (and necessary) conditions proposed in the literature; see [10] and [2].

Theorem 1: Consider (1) and (2) and assume that the pair $\{f, h\}$ is locally detectable and that

$$
\operatorname{rank}\left(\left.\frac{\partial h}{\partial x}\right|_{x=0}\right)=p .
$$

Suppose, moreover, that there exist a scalar function $V(x) \in C^{1}$, positive definite in a neighborhood of the origin, and a $m \times 1$ matrix function $G(x) \in C^{1}$ such that

$$
\begin{aligned}
0= & V_{x}(x) f(x)-\frac{1}{4} V_{x}(x) g(x) g^{\prime}(x) V_{x}^{\prime}(x) \\
& +h^{\prime}(x) h(x)+G^{\prime}(x) G(x) \\
0= & V_{x} f(x) \quad \forall x \in \operatorname{ker}(h) .
\end{aligned}
$$

Then, there exists a orthogonal matrix $T(x) \in C^{1}$ such that the function

$$
\eta(x)=T(x) G(x)-\frac{1}{2} g^{\prime}(x) V_{x}^{\prime}(x)
$$

is such that

i) for all $x \in \operatorname{ker}(h)$

$$
\eta(x)=0
$$

${ }^{1}$ This is also denoted with $h^{-1}(0)$, see, e.g., [9]. 
ii) the origin is a locally asymptotically stable equilibrium of the system $\dot{x}=f(x)+g(x) \eta(x)$

iii) the trajectories $x(t)$ of the system $\dot{x}=f(x)+g(x) \eta(x)$ starting close to the origin are such that the output $y(t)=h(x(t))$ and the control $u(t)=\eta(x(t))$ are square integrable signals.

Moreover, if

$$
p \geq k=\operatorname{rank}\left(\frac{\partial \eta(x)}{\partial x}\right) \quad \forall x \in \Omega
$$

for some constant $k$ and some neighborhood $\Omega$ of $x=0$, then

iv) in a neighborhood of the origin, $\eta(x)$ is a function of $y$, i.e. $\eta(x)=\phi(y)$ for some smooth function $\phi(\cdot)$.

Proof: Point i). By (4) and (5), it follows:

$$
\frac{1}{4} V_{x}(x) g(x) g^{\prime}(x) V_{x}^{\prime}(x)=G^{\prime}(x) G(x) \quad \forall x \in \operatorname{ker}(h) .
$$

This means that there exists a smooth orthogonal matrix $T(x)$ such that

$$
T(x) G(x)=\frac{1}{2} g^{\prime}(x) V_{x}^{\prime}(x) \quad \forall x \in \operatorname{ker}(h) .
$$

Point ii). By (6) and the orthogonality of the matrix $T(x)$, the Hamilton-Jacobi equation (4) can be rewritten as

$$
0=V_{x}(x)(f(x)+g(x) \eta(x))+h^{\prime}(x) h(x)+\eta^{\prime}(x) \eta(x) .
$$

Note now that detectability of the pair $\{f, h\}$ implies the detectability of the pair $\{f+g \eta, \operatorname{col}(h, \eta)\}$. Hence, by a classical Lyapunov argument, claim ii) holds.

Point iii). Equation (9) can be rewritten as

$$
\dot{V}=-h^{\prime}(x(t)) h(x(t))-\eta^{\prime}(x(t)) \eta(x(t)) .
$$

Hence, by local asymptotic stability of the closed-loop system, one has

$$
V(x(0))=\int_{0}^{\infty}\left(\|h(x(t))\|^{2}+\|\eta(x(t))\|^{2}\right) d t
$$

which establishes the claim.

Point iv). This claim follows from the assumptions, (8), and the rank theorem, see [9, App. A].

Remark 1: The existence of a positive-definite function $V(x)$ satisfying (4), together with the detectability of the pair $\{f, h\}$, implies the existence of state feedback control law locally asymptotically stabilizing system (1). However, unlike the case of linear systems, the existence of a locally stabilizing feedback for (1), together with the detectability of the pair $\{f, h\}$, does not imply the existence of a (locally) positive definite function $V(x)$ satisfying (4).

Remark 2: Condition (5) is trivially satisfied if $h(x)=x$, i.e., if the whole state is available for feedback.

The conditions in Theorem 1 involve two unknown, the function $V(x)$ and the mapping $G(x)$. Conditions involving only one unknown, namely only $V(x)$, can be easily derived as expressed in the following statement.

Corollary 1: Assume that there exists a scalar function $W(x) \in$ $C^{1}$, positive definite in a neighborhood of the origin, such that

$$
\begin{aligned}
0 \geq & W_{x}(x) f(x)-\frac{1}{4} W_{x}(x) g(x) g^{\prime}(x) W_{x}^{\prime}(x) \\
& +h^{\prime}(x) h(x) \\
0= & W_{x} f(x) \quad \forall x \in \operatorname{ker}(h) .
\end{aligned}
$$

Then there exist a (nonunique) $m \times 1$ matrix function $G(x) \in C^{1}$ and a scalar function $V(x) \in C^{1}$, positive definite in a neighborhood of the origin, such that (4) and (5) are satisfied.
Proof: The claim is trivially obtained setting $V(x)=W(x)$ and $G(x)$ such that

$$
\begin{aligned}
G^{\prime}(x) G(x)=-V_{x}(x) & f(x) \\
& +\frac{1}{4} V_{x}(x) g(x) g^{\prime}(x) V_{x}^{\prime}(x)-h^{\prime}(x) h(x) \geq 0 .
\end{aligned}
$$

Unlike linear systems, for which necessary and sufficient conditions are available, for general nonlinear systems only partial converse results can be derived, as expressed in the following statement, which is a partial converse of Theorem 1 . Note that a partial converse of Corollary 1 can be obtained with similar arguments.

Theorem 2: Consider (1) and (2), and assume that the pair $\{f, h\}$ is locally observable. Assume, moreover, that there exists a continuous function $\phi(y)$, with $\phi(0)=0$, such that

a) the origin is a locally asymptotically stable equilibrium of the system $\dot{x}=f(x)+g(x) \phi(y)$

b) the trajectories $x(t)$ of the system $\dot{x}=f(x)+g(x) \phi(y)$ starting close to the origin are such that the output $y(t)=h(x(t))$ and the control $u(t)=\phi(y(t))$ are square integrable signals.

Then there exist a scalar function $V(x) \in C^{1}$, positive definite in a neighborhood $\Omega$ of the origin, a $m \times 1$ continuous function $G(x)$, and an orthogonal matrix $T(x) \in C^{1}$ such that

$$
\begin{aligned}
& \text { i) } \quad 0=V_{x}(x) f(x)-\frac{1}{4} V_{x}(x) g(x) g(x)^{\prime} V_{x}(x) \\
& +h^{\prime}(x) h(x)+G^{\prime}(x) G(x) \\
& \text { ii) } \quad 0=V_{x}(x) f(x) \quad \forall x \in \operatorname{ker}(h) \\
& \text { iii) } \quad 0=T(x) G(x)-\frac{1}{2} g^{\prime}(x) V_{x}(x) \quad \forall x \in \operatorname{ker}(h) \\
& \text { iv) } \quad p \geq \operatorname{rank}\left(\frac{\partial \phi}{\partial x}\right) \quad \forall x \in \Omega \text {. }
\end{aligned}
$$

Proof: Given an initial condition $\bar{x} \in \Omega$, let $x(t)$ be the corresponding state trajectory of the system $\dot{x}=f(x)+g(x) \phi(y)$ and define

$$
V(\bar{x})=\int_{0}^{\infty}\|h(x(t))\|^{2}+\|\phi(y(t))\|^{2} d t .
$$

By assumption b) and the observability of the pair $\{f, h\}$, we conclude that $V(\bar{x})$ is positive definite and differentiable in $\Omega$. Moreover, it satisfies (9) with $\eta(x)$ replaced by $\phi(h(x))$. Set now $G(x)=\phi(h(x))+$ $(1 / 2) g^{\prime}(x) V_{x}^{\prime}$ and $T(x)=I$ and observe that points i)-iii) are trivially satisfied. Finally, claim iv) follows directly from $\phi(y)$ being a function of $y$ only.

To conclude this section, we show that, for linear systems, the conditions expressed in Theorem 1 are the nonlinear counterpart of wellknown sufficient (and necessary) conditions, as given, e.g., in [10].

Corollary 2: Consider (1) and (2). Suppose $f(x)=A x, g(x)=B$, $h(x)=C x$, the pair $\{A, B\}$ is controllable and the pair $\{A, C\}$ is observable.

The system is SOF stabilizable, by means of a linear feedback, if (and only if) there exist a symmetric positive-definite matrix $P \in \mathbb{R}^{n \times n}$ and a matrix $G \in \mathbb{R}^{m \times n}$ such that

$$
\begin{aligned}
A^{\prime} P+P A-P B B^{\prime} P+C^{\prime} C+G^{\prime} G & =0 \\
V\left(A^{\prime} P+P A\right) V & =0
\end{aligned}
$$

with $V=I-C^{\prime}\left(C C^{\prime}\right)^{-1} C$.

Proof: The proof of the sufficiency is a trivial consequence of Theorem 1. For the proof of the necessity, see [10] and [2].

\section{SIMPLE ILluSTRATIVE EXAMPLE}

In this section we present a simple example to illustrate Theorem 1. Consider a rigid body in an inertial reference frame and let $x_{1}, x_{2}$ and 
$x_{3}$ denote the angular momentum components along a body fixed reference frame having the origin at the center of gravity and consisting of the three principal axes. The Euler's equations for the rigid body with two independent controls are

$$
\begin{aligned}
& \dot{x}_{1}=\left(\frac{1}{I_{3}}-\frac{1}{I_{2}}\right) x_{2} x_{3}+u_{1} \\
& \dot{x}_{2}=\left(\frac{1}{I_{1}}-\frac{1}{I_{3}}\right) x_{3} x_{1}+u_{2} \\
& \dot{x}_{3}=\left(\frac{1}{I_{2}}-\frac{1}{I_{1}}\right) x_{1} x_{2}+\alpha u_{1}+\beta u_{2}
\end{aligned}
$$

where $I_{1}>0, I_{2}>0$ and $I_{3}>0$ denote the principal moments of inertia, $u=\operatorname{col}\left(u_{1}, u_{2}\right)$ are the control torques, and $\alpha$ and $\beta$ are two constant numbers depending on the location of the actuators. The only variable available for feedback is $y=C x$ where $C$ is a constant matrix which describes the position and the number of the sensing devices.

The problem of stabilization using dynamic feedback (either observer based or not) has been addressed in [1] and [11]. We now focus on the static output feedback stabilization problem and we show how it is possible to make use of the result in Theorem 1 . The point of departure is the consideration that for system (12) there exist two positive definite functions which are natural candidate to test the conditions in Theorem 1, namely the kinetic energy, i.e.,

$$
K(x)=\frac{1}{2}\left(\frac{x_{1}^{2}}{I_{1}}+\frac{x_{2}^{2}}{I_{2}}+\frac{x_{3}^{2}}{I_{3}}\right)
$$

and the modulo of the angular momentum vector, i.e.,

$$
P(x)=\frac{1}{2}\left(x_{1}^{2}+x_{2}^{2}+x_{3}^{2}\right) .
$$

In fact, simple calculations show that along the trajectories of (12) with $u_{1}=u_{2}=0$ one has

$$
\dot{K}=\dot{P}=0
$$

As a result, the following facts can be established.

Proposition 1: Consider (12) with output $y=C x$. Suppose that

$$
C=\left[\begin{array}{lll}
c_{11} & c_{12} & c_{13} \\
c_{21} & c_{22} & c_{23}
\end{array}\right]
$$

that $\operatorname{rank}(C)=2$ and that the system is detectable.

The origin can be made a globally asymptotically stable equilibrium of the system by means of static output feedback if there exists a pair $(\lambda, \mu)$ such that

$$
\left\{(\lambda, \mu) \mid[\lambda, \mu]^{\prime} \in \operatorname{ker} J\right\} \bigcap_{i=1,2,3}\left\{(\lambda, \mu) \mid \lambda+\frac{\mu}{I_{i}}>0\right\} \neq \emptyset
$$

where

$$
J=\left[\begin{array}{ll}
\left(\beta C_{12}-C_{13}\right) I_{2} I_{3} & \beta C_{12} I_{2}-C_{13} I_{3} \\
\left(\alpha C_{12}+C_{23}\right) I_{1} I_{3} & \alpha C_{12} I_{1}+C_{23} I_{3}
\end{array}\right]
$$

and

$$
C_{i j}=\operatorname{det}\left[\begin{array}{ll}
c_{1 i} & c_{1 j} \\
c_{2 i} & c_{2 j}
\end{array}\right]
$$

Proof: If condition (13) holds there exist $\lambda^{\star}$ and $\mu^{\star}$ such that the function

$$
V(x)=\lambda^{\star} P(x)+\mu^{\star} K(x)
$$

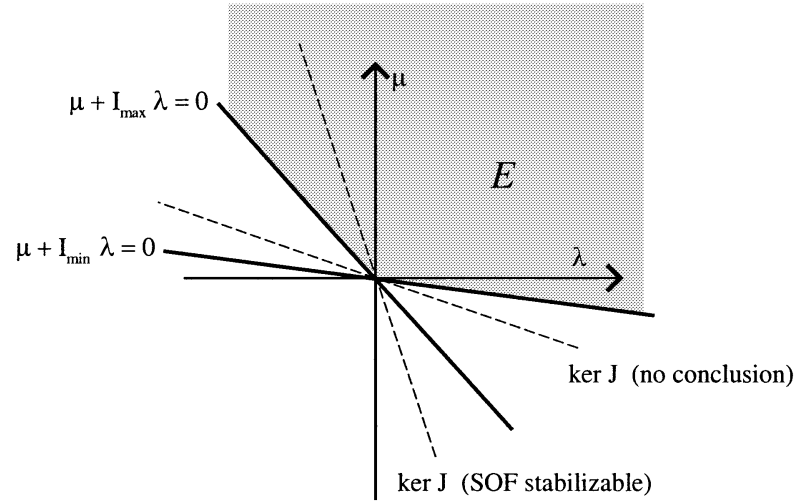

Fig. 1. Graphical representation of the sets $E$ and $\mathcal{J}$. If $\mathcal{J}=$ ker $J$ intersects the shaded region then the stabilization problem is solvable, otherwise the sufficient condition does not yield any conclusion.

is positive definite and satisfies (5). To conclude the proof, it suffices to verify that the function $V(x)$ satisfies (10) as well. For, note that with this selection of $V(x),(10)$ reduces to

$$
C^{\prime} C \leq X B B^{\prime} X
$$

where $X$ is the (constant) Hessian matrix of the function $V(x)$ and

$$
B=\left[\begin{array}{ll}
1 & 0 \\
0 & 1 \\
\alpha & \beta
\end{array}\right] \text {. }
$$

Note now that (14) implies

$$
B^{\perp} X^{-1} C^{\prime} C X^{-1}\left(B^{\perp}\right)^{\prime}=0
$$

and this holds if $\lambda^{\star}$ and $\mu^{\star}$ are in the kernel of $J$.

Remark 3: The condition expressed in Proposition 1 can be given a simple geometrical interpretation, as shown in Fig. 1. In the space $(\lambda, \mu)$, the set

$$
E=\bigcap_{i=1,2,3}\left\{(\lambda, \mu) \mid \lambda+\frac{\mu}{I_{i}}>0\right\}
$$

is a convex set, i.e., the shaded area in Fig. 1, whereas the set

$$
\mathcal{J}=\{(\lambda, \mu) \in \operatorname{ker} J\}
$$

is either a straight line through the origin, if $\operatorname{det} J=0$, or the singleton $(\lambda, \mu)=(0,0)$, if det $J \neq 0$. As a result, the sufficient condition in Proposition 1 could be easily tested. For instance, see [3], typical values for the principal moments of inertia of a small satellite are $I_{1}=27 \mathrm{~kg}$ $\mathrm{m}^{2}, I_{2}=17 \mathrm{~kg} \mathrm{~m}^{2}$, and $I_{3}=25 \mathrm{~kg} \mathrm{~m}^{2}$. Suppose now that $\alpha=0$ and

$$
C=\left[\begin{array}{lll}
1 & 0 & 0 \\
0 & 1 & \gamma
\end{array}\right] \text {. }
$$

Then, a simple calculation, show that the conditions in Proposition 1 hold if $\beta$ and $\gamma$ are such that

$$
\gamma>\frac{17}{108} \beta>0 .
$$

Proposition 2: Consider (12) with output $y=C x$. Suppose that

$$
C=\left[\begin{array}{lll}
c_{1} & c_{2} & c_{3}
\end{array}\right]
$$

that $\operatorname{rank}(C)=1$ and that the system is detectable. The origin can be made a globally asymptotically stable equilibrium by means of static output feedback if

$$
\{(\lambda, \mu) \in \Omega\} \bigcap_{i=1,2,3}\left\{(\lambda, \mu) \mid \lambda+\frac{\mu}{I_{i}}>0\right\} \neq \emptyset
$$


where

$$
\Omega=\{(\lambda, \mu) \mid \operatorname{det} \mathcal{J}=0\}
$$

and

$$
\mathcal{J}=\left[\begin{array}{ccc}
\lambda+\frac{\mu}{I_{1}} & 0 & c_{1} \\
0 & \lambda+\frac{\mu}{I_{2}} & c_{2} \\
\alpha\left(\lambda+\frac{\mu}{I_{3}}\right) & \beta\left(\lambda+\frac{\mu}{I_{3}}\right) & c_{3}
\end{array}\right] .
$$

Remark 4: As Proposition 1, Proposition 2 also admits a simple geometrical interpretation. For, note that the set $\Omega$ is either the singleton $(\lambda, \mu)=(0,0)$, or the line $\lambda=0$ if $c_{2} \beta I_{2}-c_{3} I_{3}+\alpha c_{1} I_{1}=0$, or the line $\mu=0$ if $-c_{3}+\beta c_{2}+\alpha c_{1}=0$, or two (possibly coincident) straight lines through the origin.

\section{CONCLUding REMARKS}

The problem of local static output feedback stabilization for nonlinear systems has been studied. A sufficient condition has been developed, together with a partial converse. These are a nonlinear enhancement of existing conditions for linear systems.

These results have been used to derive sufficient conditions for static output feedback stabilization of the Euler's equations with two controls and with one or two measured outputs. It is an open problem to verify if the conditions expressed in Propositions 1 and 2 are also necessary for static output feedback stabilization.

Note that the results in Propositions 1 and 2 rely on the existence of two conserved quantities, which are natural candidates to test the conditions in Theorems 1 or 2 . Therefore, it is expected that the proposed theory might be used in the design of static output feedback controllers for general Hamiltonian systems.

\section{REFERENCES}

[1] A. Astolfi, "Output feedback control of the angular velocity of a rigid body," Syst. Control Lett., vol. 36, pp. 181-192, 1999.

[2] A. Astolfi and P. Colaneri, "Static output feedback stabilization of linear and nonlinear systems," presented at the 39th Conf. Decision Control, Sydney, Australia, 2000.

[3] A. Astolfi and M. Lovera, "Global spacecraft attitude control using magnetic actuators," presented at the Amer. Control Conf., Anchorage, AK, 2002.

[4] C. I. Byrnes and A. Isidori, "Asymptotic stabilization of minimum phase nonlinear systems," IEEE Trans. Automat. Contr., vol. 36, pp. 1122-1137, Oct. 1991.

[5] C. I. Byrnes, A. Isidori, and J. C. Willems, "Passivity, feedback equivalence, and the global stabilization of minimum phase nonlinear systems," IEEE Trans. Automat. Contr., vol. 36, pp. 1228-1240, Nov. 1991.

[6] L. El Ghaoui, F. Oustry, and M. AitRami, "A cone complementarity linearization algorithm for static output-feedback and related problems," IEEE Trans. Automat. Contr., vol. 42, pp. 1171-1176, Aug. 1997.

[7] J. C. Geromel, C. E. de Souza, and R. E. Skelton, "Static output feedback controllers: Stability and convexity," IEEE Trans. Automat. Contr., vol. 43, pp. 120-125, Jan. 1998

[8] G. Gu, "Stabilizability conditions of multivariable uncertain systems via output feedback," IEEE Trans. Automat. Contr., vol. 35, pp. 925-927, Aug. 1990.

[9] A. Isidori, Nonlinear Control Systems, 2nd ed. New York: SpringerVerlag, 1989.

[10] V. Kucera and C. E. de Souza, "A necessary and sufficient condition for output feedback stabilizability," Automatica, vol. 31, pp. 1357-1359, 1995.

[11] F. Mazenc and A. Astolfi, "Robust output feedback stabilization of the angular velocity of a rigid body," Syst. Control Lett., vol. 39, pp. 203-210, 2000.
[12] V. L. Syrmos, C. T. Abdallah, P. Dorato, and K. Grigoriadis, "Static output feedback-A survey," Automatica, vol. 33, no. 2, pp. 125-137, 1997.

[13] Y.-Y. Cao, J. Lam, and Y.-X. Sun, "Static output feedback stabilization An ILMI approach,” Automatica, vol. 34, pp. 1641-1645, 1998.

\section{Optimal Containment Control for a Class of Stochastic Systems Perturbed by Poisson and Wiener Processes}

\author{
Ilya Kolmanovsky and Tatiana Maizenberg
}

\begin{abstract}
In this note, we consider a class of nonlinear stochastic systems driven by Wiener and Poisson processes. The Wiener process input enters into the equations additively to the dynamics while the Poisson process input enters into the equations multiplicatively to the control input. Examples of applied problems that may lead to system models of this kind are discussed in the note. The optimal containment control problem is then formulated for these systems. It involves either maximizing the time of stay within an admissible set or a closely related performance measure. The optimal control and the optimal value function are characterized on the basis of Bellman's dynamic programming principle in the general case so that the optimal value function is a solution of a boundary value problem for a partial differential equation (PDE). For a special case defined by more restrictive assumptions the method of successive approximations is used to show the existence of solution to this boundary value problem and to set up an iterative solution procedure. An example is reported that illustrates the results.
\end{abstract}

Index Terms-Dynamic programming, jump-diffusion, optimal control, Poisson process, stochastic control.

\section{INTRODUCTION}

Differential equations driven by Poisson processes arise quite frequently as models in engineering, manufacturing, and economics applications. They may represent a discontinuous change in the state of the system caused by significant abnormal events. One may recall that a trajectory of a Poisson process $\eta(t)$ either stays at a constant value or undergoes jumps. The jump size is equal to 1 while the number of jumps in the time interval of length $\Delta t$ is distributed according to the Poisson law

$$
\operatorname{Prob}\{\Delta \eta(t) \equiv \eta(t+\Delta t)-\eta(t)=k\}=e^{-\lambda \Delta t} \frac{(\lambda \Delta t)^{k}}{k !} .
$$

Thus, during a small time interval of length $\Delta t$ the process may jump upward with the probability approximately equal to $\lambda \Delta t$. With the probability approximately equal to $1-\lambda \Delta t$ the process stays at a constant value. In manufacturing systems, the Poisson processes may represent the effects of the machine breakage or repair [9]. In inventory systems, the arrival of new customers, increase in demand, the arrival of supply or new resource that allows to replenish the inventory can be modeled using Poisson processes [3]. In finance and economics applications, Poisson processes are used to model the effect of rare but important events on asset prices [6].

Manuscript received June 7, 2001; revised December 19, 2001 and July 15, 2002. Recommended by Associate Editor X. Zhou.

I. Kolmanovsky is with Ford Research Laboratory, Ford Motor Company, Dearborn, MI 48124 USA (e-mail: ikolmano@ford.com).

T. Maizenberg is with Moscow State Mining University, Moscow, Russia Digital Object Identifier 10.1109/TAC.2002.805679 